\title{
Digitising Shop Floor Visualisation Boards: A Missing Link in the Industry 4.0 Era
}

\author{
John Bang MATHIASEN ${ }^{1 \mathrm{a}}$ and Pernille CLAUSEN \\ Aarhus University, Department of Business Development and Technology, Denmark
}

\begin{abstract}
This paper aims at conceptualising a digitised Shop Floor Management (SFM) visualisation board. The logic of inquiry throughout the study is an iterative back and-forth approach between our theoretical conceptualisation of the digitised visualisation board and empirical data collected in three industrial companies. The paper shows that digitised visualisation boards should have malleable representation capacities to transfer, translate and transform knowledge within and across SFM teams. A digitised visualisation board is suggested, which consists of; translating practices within SFM teams, translating practices across SFM teams, transforming practices across SFM teams and translating practices within SFM teams.
\end{abstract}

Keywords. Shop Floor Management, Digitisation, Visualisation, Sharing Knowledge.

\section{Introduction}

Shop Floor Management (SFM) visualisation boards are instrumental in enabling performance management (PM) and continuous improvement (CI) activities, e.g. [1]. By its nature, SFM decision-making unfolds as social interactions [2] guided by the practitioners" "conversation with" the visualisation board [3]; the representation capacities of the visualisation boards [4] are the glue for the social interactions [5].

At the threshold of the Industry 4.0 (I4.0) era in which the complexity of decisionmaking within SFM practices is expected to increase [6], it seems relevant to study the representation capacities of SFM visualisation boards, for instance; what role(s) should visualisation boards have?, and what kind of representation fidelity should visualisation boards have? Actually, the design and thus representation capacities of the prevalent SFM visualisation boards originate from the principles of Toyota Production System [7] and thus Lean [8]. These principles highlight the power by pen entailing the use of nondigitised whiteboards rather than software-based systems [9], easy to understand information [10], and the importance of having brief rather than lengthy SFM meetings [11]. However, the non-digitised visualisation boards, a leftover from the Industry 2.0 (I2.0) era [12], do not enable the application of real-time data, reliable data and big data in the decision-making activities. In addition, the non-digitised visualisation board entails that data and information remain in functional silos [13], which complicates transdisciplinary decision-making within SFM practices.

Recently, academia has problematized the use of non-digitised boards as visualisation tools, mainly due to the exponential development of digitised technologies [14], big data

${ }^{1}$ Corresponding Author, Email: johnbm@btech.au.dk. 
[15] and artificial intelligence [16] as these technologies will open up new possibilities for improving decision-making activities within SFM practices [17]. To gain an understanding of accomplishing shop floor management activities in the I4.0 era, this paper aims at conceptualising a digitised visualisation board and suggest some of its managerial opportunities.

To answer the research question "what kind of representation capacities should a digitised visualisation board have?" we draw upon three cases, which shed light on SFM activities in three different Danish companies. The three cases explicate the characteristic of SFM activities in terms of using visualisation boards to accomplish PM and CI activities. By juxtaposing the empirical findings from the three cases with related theories, we conceptualise a framework for a digitised visualisation board, which has different representation capacities.

The paper's contributions are; a conceptualised model of digitised visualisation boards having malleable representation capacities to guide different kinds of PM and CI activities: and that the representation capacities of the digitised board unfold when handling PM and CI activities: and that this situated base model is instrumental in conveying meaning within and across SFM boundaries.

In the following, we present the theoretical background of the study including a preliminary version of the framework for the digitised SFM visualisation board, followed by the methodological considerations and the presentation of the three cases. Next, we revise the preliminary framework, present the discussion and conclusion of the paper.

\section{Theory}

Drawing on the work of $[4 ; 5 ; 7 ; 18 ; 19]$ this paper defines SFM visualisation boards as material entities, which have representation capacities to influence the practitioners' understanding when accomplishing PM and CI activities. This definition implies that practitioners' "conversation with" the visualisation board [3] pave the way for converting data and information into visual meaning at the individual level [5], and this individual visual meaning into common understanding among the involved practitioners [18], which enables transdisciplinary decision-making.

I4.0 in a manufacturing context can be understood as a digital transformation that has reshaped the manufacturing equipment at SFM level to bring smart manufacturing on the forefront [20]. This digitised equipment generates big data and reliable real-time data and combined with intelligent systems [16] it becomes possible to utilise the predictive power of data. However, companies are only less capable in retrieving and visualising data, which obstructs practitioners to convey information and knowledge sharing at SFM level [7]. Likewise, the fundamental design of SFM visualisation boards can be tracked back to mid-1940s [7] entailing that the SFM practices [5] is stuck in the I2.0 era [21].

Digitised visualisation boards are available, but due to the advices of having short stand-up meetings [22], "power by the pen" rather than digitised systems [9] and simplicity in the visualisation of data [7], only few companies are using digitised visualisation boards [12]. Likewise, in the I4.0 era the complexity of managing PM and CI increases [6], which necessitates that decision-making within SFM practices will be a transdisciplinary activity with practitioners cutting across functional and organisational boundaries [19]. The question is whether the available digitised visualisation boards have the required representation capacities to function as an effective and efficient tool for practitioners when they are involved in decision-making activities. 
SFM visualisation boards have gained huge attention from academia as instrumental in accomplishing SFM activities, which can have different degree of complexity [10]. In the scope of this paper, academia has improved our understanding of the roles of visualisation boards to provide a common understanding. This necessitates highly visual information [1], to display "easy-to-see" and "easy-to-read" information and requirements [23], as well as in setting directions and guiding actions [7], in facilitating two-way-communications [24], and in conveying information and knowledge sharing [5] within and across different functional boundaries [25]. The fidelity of the representation displayed at visualisation boards should support situated decision-making [18], meaning that useful representations can be abstract or concrete and thus mutable rather than static [4]. Hence, companies should avoid a "one-size fits all" approach [9].

In general, the representation capacities of visualisation boards are in the literature conceptualised as abstract (low fidelity) or concrete (high fidelity) and as used within or across practices $[4 ; 19]$. To study what kind of representation capacities a digitised visualisation boards should have this paper focuses on the fidelity of the representations as a low-high fidelity dimension and on the role of the representations in terms of enabling within-across knowledge sharing dimension. Before addressing the role and fidelity of visualisation boards, SFM decision-making is discussed.

The complexity of SFM decision-making has been discussed by Liker and Meyer [10] who suggest three degrees of complexity; from simple issues occurring repeatedly throughout the day, medium issues influencing the SFM team or department, and large issues having an organisational effect. Hertle et al. [26] draw on this taxonomy and suggest that decision-making of simple issues is handled directly by the practitioners, the team manager should evaluate medium issues and subsequently the SFM team handles decision-making, while large issues decision-making should be escalated across SFM practices involving management and transdisciplinary problem solving activities. However, in the I4.0 era, it is expected that interconnection and interoperability among technical equipment [15] and artificial intelligence [14], result in a situation where both the social- and technical system are involved in the decision-making at SFM level.

The decision-making conducted by the digitised equipment (technical system) has limitations compared to the human-based decision-making. It is unlikely that technical systems exceed practitioners in the near future; in contrast to computer-controlled equipment, practitioners have common sense [27], creative intelligence, social intelligence as well as perception and manipulation capabilities to accomplish decisionmaking in unstructured and changing contexts [28]. Hence, drawing on the work of [6; 19 ; 29] this paper suggests that decision-making of simple issues will be handled by digitised equipment, but practitioners will be involved in two types of decision-making; either decision-making within SFM practices, or decision making involving practitioners cutting across SFM boundaries. In the next section, we address the role of the visualisation boards in relation to knowledge sharing within and across SFM boundaries.

The role of digitised visualisation boards are essential for knowledge sharing within SFM practice [5]. In relation to decision-making cutting across SFM boundaries, it is crucial that the involved practitioners gain access to and can utilise knowledge embedded in other practices [19], for instance by applying digitised visualisation boards [17]. In other words, decision-making goes hand in hand with knowledge sharing [30].

The Deweyan pragmatism [31] suggests that the formation of new knowledge is individualised, but a human's formation of knowledge does also involve social interaction, see [32]. Drawing on the work of Schön's pragmatism [3] knowledge sharing unfolds as a process of social interaction in which practitioners have "reflective 
conversation with" the visualisation boards. Ewenstein and Whyte [4] suggest that a plurality of social interactions unfolds around the visualisation board. For instance, when accomplishing CI various professional competencies such as industrial engineers, technicians, team managers and blue-collar workers are involved in the "reflective conversation with" the visualisation board. Despite these practitioners might have different intentions [33], might come from different "object worlds" in which practitioners speak different languages [34], and might have different understandings of the data and information displayed at the visualisation board [18], the visualisation board is common enough through rules, common frames of reference and shared archives to make them recognizable as means of translation [35] and in gaining access to apply knowledge embedded in other practices [25]. However, because of this embeddedness of knowledge in practice, a one-size-fits-all visualisation board to facilitate knowledge sharing is problematic; the next section addresses this.

The fidelity of digitised visualisation boards should facilitate all involved practitioners to distinguish between normal and abnormal situations at glance [7] and to watch the performance of the SFM in relation to established key performance indicators [11]. In the same vein, Tao et al. [15] suggest having a real-time virtual SFM, which plays the same important role as the physical SFM practice does. In contrast to these advocates for high fidelity visualisation boards, other researchers highlight that SFM practitioners are involved in handling a large variety of tasks such as CI having different complexity [26], which often calls for transdisciplinary collaboration. These researchers suggest applying context-sensitive and low fidelity visualisation boards [18] to gain benefit from the power by hand principles [9] and thus flexible problem-solvers [36].

Because of doing SFM ranks from simple monitoring the situation to complex transdisciplinary decision-making, the knowledge to be shared via the digitised visualisation board will have various nature [37]. It entails that the "transfer" of knowledge is not straightforward; indeed, "to transfer is to translate or to transform" [38]. To sum up, visualisation boards are the focal point for knowledge sharing and it should have sufficient representation capacities to enable different kinds of decision-making. A group of researchers draws on American pragmatism, e.g. [25] to make a distinction between knowledge having a syntactic nature, which is directly understandable; knowledge having a semantic nature and due to the lack of a formal syntax, interpretation is required; and pragmatic nature of knowledge calls for communication and negotiation. This paper subscribes to the viewpoint that the representation capacities of the digitised visualisation boards should enable the transfer of syntactic knowledge, the translation of semantic knowledge and transformation of pragmatic.

\section{Methodological considerations}

The paper draws on Stake's [39] collective instrumental case study of three Danish companies, all operating globally; each of the three companies is a particular case providing insight into answering the research question "what kind of representation capacities should a digitized visualisation board have?". Focusing on the use of and requirements to visualisation boards in the three companies seems appropriate given that we want to gain an empirical understanding of SFM decision-making and representation capacities of visualisation boards in different contextual settings. The analysis in the paper is an iterative back and-forth approach between theory and empirical data. This abductive approach [40] was useful as it paved the way for moving between the empirical 
data and our preliminary conceptualisation to explore the representation capacities of digitised visualisation boards. To enhance the trustworthiness of the study we discussed our observations and interviews with the involved practitioners in each of the three companies. The data collection consists of observations in which one of the authors took the role as complete observer and conducted the semi-structured interviews. In average, each observation lasted two hours in which notes were taken. Each interview lasted in average 30 minutes and notes were taken simultaneously. In line with the abductive approach, both the observations and interviews were guided by our gradually increased empirical understandings and theoretical conceptualisation. The three case-companies have be designated Company A, B and C. Company A, a global leader in the renewable energy industry has 23.000 employees; four SFM board meetings are observed and three interviews are conducted. Company B, a brewery has 40.000 employees; two SFM board meetings are observed and two interviews are conducted. Finally Company $\mathrm{C}$, operating in the meat processing industry has 26.000 employees; two SFM board meetings are observed and two interviews are conducted.

\section{Cases}

Company $A$ conducts SFM meetings every day, which takes in average 20 minutes; handmade notes are taken and written down on A4-paper. Several performance indicators are discussed and potential issues are handled within this short time-frame; thus, the meetings are very efficient. The company applies both analogue and digitised visualisation boards, but at presented, the company does mainly apply manually updated analogue visualisation boards. Due to a very complex production set-up and the use of semi-automatized production equipment, it is crucial that all workstations (SFM practice) comply with the fixed takt-time; in this regard, break-downs of equipment, lack of materials and quality issues are problematics for planning the production. To handle all these performance issues the company has initiated the implementation of digitised visualisation boards as support tools in decision-making related to enhance the efficiency of the production. Accordingly, the digitised visualisation boards, which display takttime information and strategic established key performance indicators enable data transparency across all workstations and are applied to solve potential problematic performance issues. The company has realised an ongoing need for enhancing the data foundation for decision-making at SFM level and as explained in the above, they have already invested many resources in implementing digitised visualisation boards. In addition to support different kinds of decision-making, the company can see benefits in capturing notes from SFM meetings digitally. Given that all practitioners involved in the current SFM board meetings are highly engaged and proactive, company A believe they are on the right track in terms of future implementation of digitised visualisation boards.

Company $B$ accomplishes SFM meetings every day, which in average lasts for 20 minutes. At present, the majority of the SFM board meetings applies manually updated analogue boards. As for the board meetings being observed, all SFM practices in the brewery are represented. Each of these practitioners accounts for performance since the last SFM meeting and a give situational statement of the SFM practice in question. The objective is to form SFM practices in which the practitioners proactively share knowledge and thereby increase the performance level. However, at present the practitioners describe the SFM meetings as being inefficient; indeed the practitioners consider the current approach for doing SFM meetings as a consequence of following the standard procedures strictly. In the same way, the company has realised that the 
practitioners' current reactive approach during the SFM meetings is caused by a lack of using real-time data and too unstructured data for analytical decision-making. Accordingly, the management has realised an urgent need for improving the SFM meetings. In the same vein, because of strict legalisation requirement in the food industry and a need of monitoring data more frequently, a few number of SFM practices in the brewery have started using digitised visualisation boards. The application of these digitised boards has only had minimal influence on the SFM performance. To gain more benefits of using the digitised visualisation boards the company is working on enhancing information transparency across the brewery and as instrumental in capturing meetings and continues improvements notes digitally.

Company $C$ does only apply manually updated analogue boards. The SFM meetings last for around 30 minutes and are held every two weeks. The focal point for the SFM meetings is discussions and updates of CI activities in all teams across the factory; the complexity of the problems being handled are medium to high. It entails that PM activities do only gain minor attention. Because of unsuitable data foundation for collecting the necessary production/performance information, the analogue boards function mainly as bulletin boards, on which notes are written down and sketches are made to explain ideas/suggestions to solutions. Despite conclusion are made during these meetings, information about the solutions to handle problematic issues is not stored. The takt-time of the lines sets the pace for the highly standardised manufacturing activities, which means that the practitioners have to follow rather strict procedures. However, the current lack of information transparency across the factory results in planning losses. To handle the lack of information transparency the company has started an investigation of implementing digitised data treatments and analytical tools as for instance digitised visualisation boards. According to the practitioners being interviewed, a digitisation of the data treatment systems combined with a visualisation of appropriate information will have a positive influence on performance within all SFM practices.

\section{Analysing the representation capacity of the digitised board}

In an abductive manner, we juxtapose the preliminary conceptualisation presented in the theoretical section with the three cases in the preceding section to answer, "what kind of representation capacities should a digitised visualisation board have". As it appears from the preliminary conceptualisation, the framework consists of a high-low fidelity continuum and a knowledge sharing within-across boundary continuum. This conceptualisation results in four different SFM practice; transferring practice within SFM boundaries, translating practice across SFM boundaries, translating practice within SFM practices, and finally transforming practice across SFM boundaries. The different practices are elaborated in the following.

The transferring practice within the SFM boundaries enables knowledge sharing to managing performance and detecting abnormalities. Due to the dialogues about actual performance and potential abnormalities should be handled in a short time-frame, efficient transfer of easy-to-see and easy-to-read information is the focal point. The cases demonstrate that practitioners discuss various performance indicators. For instance, the performance issues in relation to malfunctions of technical equipment, lack of materials, etc. are normally handled in a short time-frame to comply with "takt-time" requirements. This means that practitioners should be capable of distinguishing between normal and abnormal performance at glance, which necessitates syntactic representation capacities. This requires high information fidelity and a common understanding of the information 
syntax depicted on the digitised boards. Thus, the digitised visualisation board should depict real-time information, which is easy understandable for all practitioners involved.

The translating practice across SFM boundaries focuses on knowledge sharing to coordinate performance issues as well as to gain commitment from and report actual SFM performance to the management or other external practitioners. The coordination of performance issues across SFM practices is central for case-company A and B; while company A conducts coordination four times a day company $\mathrm{B}$ does it once a day. To avoid misunderstandings both companies put huge efforts in coordinating performance issues as for instance lack of material or technical malfunctions. Likewise, to sustain or enhance commitment from the management all three case-companies are requested to report actual performance of strategically determined indicators. The lack of information transparency complicates the knowledge sharing across the SFM practices. Likewise, practitioners belonging to different practices have different intentions and they have different educational and practical background, which results in a lack of common syntax to understand directly the information being displayed at the digitised visualisation board. Hence, the representation capacities should be semantic and thus afford practitioners to interpret the information depicted on the digitised visualisation boards differently, i.e., knowledge sharing has to be translated.

The translating practice within SFM boundaries facilitates knowledge sharing of medium complex problems and in implementing solutions. The case-companies are forced to cope with different kinds of technical malfunctions, lack of material, and planning- and quality issues. The variety of the problems to be handled in each of the companies is broad and often the decision-making is not straightforward. The decisionmaking is often a trade-off between pros and cons, which entails that practitioners should interpret various viewpoints. The implementation of digitised visualisation boards in company $\mathrm{A}$ and $\mathrm{B}$ is done in an attempt to improve transparency and thereby enhance the accessibility of various information. Likewise, these two companies acknowledge a need of capturing notes and information digitally for both PM and CI decision-making. Having access to various kinds of information are expected to enhance decision-makings of the medium-complex problematic issues. Hence, the representation capacities of the digitised visualisation board should facilitate the involved practitioners to interpret various kinds of information, which can have a syntactic- or semantic nature. The variety of information entails that practitioners often face situations in which interpretations are required to facilitate knowledge sharing.

The transforming practice across SFM boundaries pave the way for handling highly complex problems and transdisciplinary activities. The three case-companies acknowledge a need of establishing a more appropriate data foundation. By doing so, the companies expect enhancing the transdisciplinary activities in terms of accomplishing highly complex decision-makings. To establish the data foundation both company A and $\mathrm{B}$ have invested in digitised visualisation boards, but it has only had a minor influence on solving complex problems. A lack of information transparency combined with too unstructured data complicates the transdisciplinary decision-making in these two companies, which are characterised by practitioners cutting across functional and organisational boundaries. Company $\mathrm{C}$ and partly company A handle these highly complex problems by writing notes and drawing sketches to explain and advocate for ideas/suggestions when solving these. Accordingly, the handling of highly complex decision-making involving transdisciplinary activities requires sufficient representation capacities of the information depicted on the digitised visualisation board to facilitate communication and negotiation across professional boundaries. A context-sensitive and 
low fidelity digitised visualisation board enable practitioners to share pragmatic knowledge across professional boundaries; i.e., knowledge embedded in practice and localised around the problem to be handled.

\section{Discussion and conclusion}

Figure 1 illustrates the proposed framework for digitising SFM visualisation board.

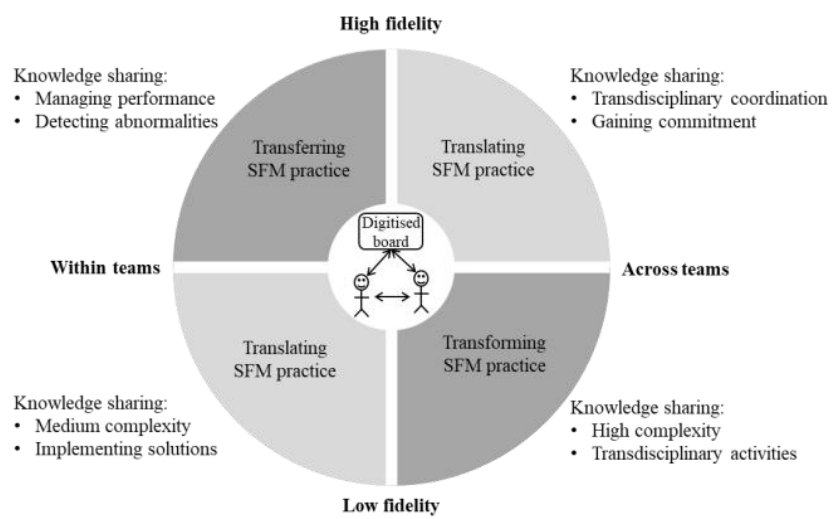

Figure 1. The digitised visualisation board.

Subscribing to pragmatism $[3 ; 25 ; 31 ; 32]$ entails that knowledge sharing, i.e., learning is an individual process, but the learning of each individual does also involve social interactions among practitioners. Accordingly, the circle in the middle of figure 1 illustrates that the practitioners" "reflective conversation with" the representation capacities of the digitised visualisation board influence both their social interaction and each of the involved practitioners' individual knowledge creation process. The analysis shows that the nature of knowledge to be shared via the digitised visualisation boards is situational; e.g., practitioners in the companies are involved in various activities. These activities rank from monitoring normal/abnormal "takt-time" performance at glance within and across SFM practices to CIs having different complexity in which practitioners are in a need of gaining access to real-time and more analysable data from external technicians. Because of this situational decision-making and different knowledge being shared, it is pivotal that the representation capacities are malleable.

As depicted in figure 1 the proposed framework to convey meaning within and across boundaries consists of two continuums: low and high fidelity as one continuum and a knowledge sharing within and across SFM boundaries as the other continuum. It entails that the representation capacities of the digitised visualisation board should enable transferring-, translating- or transforming practices for the knowledge sharing processes. The representation capacities enabling transferring practices within teams have high fidelity. Practitioners within the SFM team are familiar with the habitual way of managing performance and detecting abnormalities entailing that they have a common understanding of the information syntax. Hence, the information depicted on the digitised visualisation board should be directly understandable. As for the translating practice across teams, the transdisciplinary practitioners lack a common understanding of the habitual way of working; they might have different educational and practical background and thus speak different professional languages. In other words, a common syntax to understand the information across teams does not exist. To coordinate 
performance issues across teams and to gain commitment from and report actual SFM performance to the management the fidelity of the information should be high but also malleable. Hence, the representation capacities of the digitised visualisation boards should be semantic, which enables practitioners to translate the displayed information across teams. The transforming practices across teams is necessary to handle CIs having high complexity. Handling high complexity CIs tasks entails that the habitual way of working is really at stake. This means that the focal point for these complex transdisciplinary activities is to share knowledge, which is "localised, embedded and invested in a situated practice" [25]; it is pragmatic. To share pragmatic knowledge across professional boundaries the representation capacities of the information depicted on the digitised visualisation board should be context-sensitive and having low fidelity. Finally, the translating practice within teams unfolds when CIs have medium complexity and when implementing solutions. The habitual way of working has to be changed, but the team has sufficient knowledge to accomplish the task by themselves. The new way of working entails that practitioners have to gain new knowledge of for instance new standard procedures and to use new technological equipment. Hence, the representation capacities of the digitised visualisation boards should enable practitioners to translate and thus make the information understandable within the team to adapt the new practice.

The findings in this paper support the viewpoint that companies should avoid a "onesize fits all" approach to the design and application of visualisation boards [9] and that the fidelity of the representations can be high and low and thus mutable [4]. We suggest however, that a digitised visualisation board has to be malleable to comply with the I4.0 era. Thus, in contrast to Meissner et al. [17] who propose that the requirements for successful digitised SFM are basically the same as for existing analogue SFM, this paper demonstrates that the representation capacities of digitised boards have to adaptable to the specific problematic issues being handle within or across SFM teams. This will enable SFM practitioners and top management to gain full benefits from the exponential development of digitised technologies [14], big data [15] and artificial intelligence [16]. A digitisation of SFM practice will open up new possibilities for improving PM and CI activities within SFM practices, which can give manufacturing a more proactive role in the strategic development of the company.

The purpose of this paper was to conceptualise a digitised visualisation-board and to suggest some of its managerial opportunities. The paper suggests that the representation capacities of the digitised visualisation boards should enable; transfer of syntactic knowledge within SFM teams, the translation of semantic knowledge within as well as across SFM teams, and transformation of pragmatic knowledge across SFM teams.

\section{References}

[1] N. Bateman, L. Philp, and H. Warrander, Visual management and shop floor teams - development, implementation and use. Int. J. of Production Research, 2016, Vol. 54(24), pp. 7345-7358.

[2] B.A. Bechky, Sharing Meaning Across Occupational Communities: The Transformation of Understanding on a Production Floor. Organization Science, 2003, Vol. 14, No. 3, pp. 312-330.

[3] D.A. Schön, The Reflective Practitioner, Basic Books, Inc., Publishers, New York, 1983.

[4] B. Ewenstein and J. Whyte, Knowledge Practices in Design: The Role of Visual Representations as 'Epistemic Objects'. Organization Studies, 2009, https://doi.org/10.1177/0170840608083014.

[5] G.D. Galsworth, Visual Workplace-Visual Thinking Creating Enterprise Excellence through the Technologies of the Visual Workplace, Second Edition. Productivity Press, New York, 2017.

[6] H. Kagerman, J. Helbig, A. Hellinger and W. Wahlster, Securing the Future German Manufacturing Industry: Recommendations for Implementing the Strategic Initiative Industry 4.0, acatec, 2013. 
[7] Y. Eaidgah et al., Visual management, performance management and continuous improvement, A lean manufacturing approach. Int. J. of Lean Six Sigma, 2015, Vol. 7, No. 2, pp. 187-210.

[8] M. Imai, Kaizen: the Key to Japan's Competitive success. Random House, New York, 1986.

[9] G.C. Parry and C.E. Turner, Application of lean visual process management tools, Production Planning \& Control, 2006, Vol. 17, Issue 1, pp. 77-86.

[10] Liker, J. K., and Meier, D. (2006). The Toyota Way Fieldbook. McGraw-Hill, New York.

[11] M.V. Iuga, Visual Communication in lean organization. MATEC Web of Conferences 121, 02005 (2017).

[12] P. Clausen, J.B. Mathiasen and J.S. Nielsen, Barriers and enablers for digitizing Shop Floor Management boards: A Human Bond Communication perspective. The 6th Global Wireless Summit (GWS -2018).

[13] F. Werner and R. Woltsch, Data Processing in Industrie 4.0, Datenbank Spektrum, 2018, pp. 15-25.

[14] C. Zhuang, J. Liu and H. Xiong, Digital twin-based smart production management and control framework for the complex product assembly shop-floor, Int. J. Adv. Manuf. Techn. ,2018, Vol. 96:1, pp 1149-1163.

[15] F. Tao et al, Digital Twin Shop-Floor: A New Shop-Floor, IEEE Access, Vol. 5, 2017, pp. 20418 - 20427.

[16] K. Rajan and A. Safiotti, Towards a science integrated of AI and Robotics. Artificial Intelligence, 2017, Vol. 247, pp. 1-9.

[17] A. Meissner, M. Müller, A. Herman et al. Digitalization as a catalyst for lean production: A learning factory approach for digital shop floor management. Procedia Manufacturing, 2018, Vol. 23, pp. 81-86.

[18] P. Beynon-Davies and R. Lederman, Making sense of visual management through affordance theory. Production Planning \& Control, 2017, Vol. 28, Issue 2, pp. 142-157.

[19] S. Winby and S.A. Mohrman, Digital Sociotechnical System Design. The Journal of Applied Behavioral Science, 2018, https://doi.org/10.1177/0021886318781581.

[20] Y. Yin, K. Stecke and D. Li, The evolution of production systems from Industry 2.0 through Industry 4.0. Int. J. of Production Research, 2018, Vol. 56, Issue 1-2, pp. 848-861.

[21] M. Holm, The future shop-floor operators, demands, requirements and interpretations. Journal of Manufacturing Systems, 2018, Vol. 47, pp. 35-42.

[22] M.V. Iuga and L.I. Rosca, Comparison of problem solving tools in lean organizations. MATEC Web of Conferences 121, 02004 (2017).

[23] B. Tezel, L. Koskela et al., The functions of visual management. University of Salford, 2009.

[24] Y. Liao, F. Deschamps, E. de Loures and L.F. Ramos, Past, present and future of Industry 4.0 - a systematic literature review and research agenda proposal. Int. J. of Prod. Research, 2017, Vol. 55, pp. 3609-3629.

[25] P.R. Carlile, Transferring, Translating, and Transforming: An Integrative Framework for Managing Knowledge Across Boundaries. Organization Science, 2004, Vol. 15, No. 5, pp. 555-568.

[26] C. Hertle et al., The next generation shop floor management - how to continuously develop competencies in manufacturing environments. The 23rd Int. Conference on Production Research, Manila, 2015.

[27] E. Davis and G. Marcus, (2015). Common sense Reasoning and Common sense Knowledge in Artificial Intelligence. Communications of the ACM. Sep 2015, Vol. 58, Issue 9, pp 92-103.

[28] C.B. Frey and M. Osborne, The future of employees: how susceptible are jobs to computerisation? Oxford Martin Programme on Technology and Employment, University of Oxford, 2013.

[29] S.V. Buer, J.O. Strandhagen and F. Chan, The link between Industry 4.0 and lean manufacturing: mapping current research and establishing a research agenda. Int. J. of Prod. Res., 2018, Vol. 56, pp. 2924-2940.

[30] J. Lave and E. Wneger, Situated learning: Legitimate peripheral participation. Cambridge University Press, Cambridge, 1991.

[31] J. Dewey, Logic: The Theory of Inquiry, Southern Illinois University Press, Carbondale, 1938.

[32] H. Blumer, Symbolic interactionism: Perspective and method. University of California Press, 1969.

[33] J.B. Mathiasen, Doing product development activities: the role of experience and ends-in-view, Int. J. of Innovation and Learning, 2017, https://doi.org/10.1504/IJIL.2017.087494.

[34] L.L. Bucciarelli, Between thought and object in engineering design. Design Stud. 2002, 23:3, pp. $219-231$.

[35] S.L. Star and J. Griesemer, Institutional ecology, translation and boundary objects. The science studies reader, 1999, pp. 505-524.

[36] B. Mrugalska and M.K. Wyrwicka, Towards Lean Production in Industry 4.0. Proc Engin, 2017, 466-473.

[37] J. Stjepandić, W.J.C. Verhagen, H. Liese and P. Bermell-Garcia, Knowledge-Based Engineering. In: J. Stjepandić et al. (eds) Concurrent Engineering in the 21st Century: Foundations, Developments and Challenges, Springer International Publishing, 2015, pp. 255-286.

[38] S. Gheradi and D. Nicolini, To Transfer is to Transform: The Circulation of Safety Knowledge, Organization, 2000, https://doi.org/10.1177/135050840072008.

[39] R.E. Stake, The Case Study Method in Social Inquiry. In R. Gomm, M. Hammersley and P. Foster (eds.), Case Study Method: Key Issues, Key Texts, SAGE Publications, London, 2000, pp. 19-26.

[40] A. Dubois and L.-E. Gadde, Systematic combining: an abductive approach to case research. Journal of Business Research. Vol. 55, Issue 7, July 2002, pp. 553-560. 